\title{
Expression and clinical significance of miRNA-145 and miRNA-218 in laryngeal cancer
}

\author{
LIBING GUO $^{1}$, XIUYU CAI ${ }^{2}$, WEIHAN HU ${ }^{2}$, WENFENG HUA ${ }^{1}$, \\ WEN YAN ${ }^{1}$, YUCHENG LIN ${ }^{1}$, SHENGSONG YIN ${ }^{1}$ and YUE CHEN ${ }^{2}$ \\ ${ }^{1}$ Department of Oncology, Guangdong Second Provincial General Hospital, Guangzhou, Guangdong 510317; \\ ${ }^{2}$ Department of Radiation Oncology, Sun Yat-sen University Cancer Centre, Guangzhou, Guangdong 510060, P.R. China
}

Received January 14, 2019; Accepted April 25, 2019

DOI: $10.3892 / 01.2019 .10353$

\begin{abstract}
Expression of miRNA-145 and miRNA-218 in serum of patients with laryngeal cancer and the relationship between them and the clinicopathological parameters and prognosis were investigated. The clinical medical records of 132 patients with laryngeal cancer, who were admitted to Guangdong Second Provincial General Hospital from February 2009 to March 2014, were retrospectively analyzed and comprised the study group. The data of physical examinations of 56 healthy volunteers who took physical examinations in the same hospital comprised the control group. RT-qPCR was used to detect the expression levels of miRNA-145 and miRNA-218 in serum of the patients in the two groups. According to the relative expression levels of miRNA-145 and miRNA-218 in serum of the patients in the study group on the day when they left hospital, the study group was divided into the high expression group ( $\mathrm{n}=73$ patients) and the low expression group ( $\mathrm{n}=59$ patients). The patients received a 48 -month follow-up visit and their survival condition was recorded and the Kaplan-Meier was used to carry out the survival analysis. The expression levels of miRNA-145 and miRNA-218 in serum of the patients in the study group were lower than those in the control group $(\mathrm{P}<0.05)$. The median survival time of the patients in the high expression group was 30 months while the median survival time of the patients in the low expression group was 26 months. The expression levels of miRNA-145 and miRNA-218 in serum of patients with laryngeal cancer decreased, the higher the expression levels of miRNA-145 and miRNA-218 in serum of patients with laryngeal cancer were, the better the prognosis was. miRNA-145 and miRNA-218 were used as indicators of assessing the prognosis of patients with laryngeal cancer.
\end{abstract}

Correspondence to: Dr Libing Guo, Department of Oncology, Guangdong Second Provincial General Hospital, 466 Xingangzhong Road, Haizhu, Guangzhou, Guangdong 510317, P.R. China

E-mail: bvs2hr@163.com; goodluck.glibing@163.com

Key words: miRNA-145, miRNA-218, laryngeal cancer, prognosis, clinical significance

\section{Introduction}

Head and neck cancer is the fifth most common cancer in the world and is the most common cancer of the head and neck cancers. According to research reports in northeastern Iran, laryngeal cancer accounts for $46.70 \%$ of all head and neck cancers (1). The pathological type of laryngeal cancer is mainly squamous cell carcinoma. Studies have shown that the morbidity of laryngeal cancer is increasing $(2,3)$. In the United States, the increase of new patients with laryngeal cancer total 12,000 cases each year. Smoking and drinking are the main factors that cause laryngeal cancer; $26.60 \%$ of patients have human papillomavirus infection, the clinical symptoms of laryngeal cancer are mainly hoarseness, cough, and airway obstruction. If patients with early laryngeal cancer are treated timely, the survival rate can reach $56-93 \%$, but the survival rate of patients with advanced laryngeal cancer is only $29-56 \%$ (4). It can be seen that the early detection and early treatment are very important to improve the survival rate of patients with laryngeal cancer. The early symptoms of laryngeal cancer are not obvious, so it is usually diagnosed at a late stage, causing treatment delay and patients miss the best surgery period, which leads to a higher recurrence rate of patients with laryngeal cancer (5). The main treatment methods of laryngeal cancer are surgical treatment and radiotherapy. In recent years, with the development of technology, carbon dioxide laser laryngeal cancer surgery has become one of the main minimally invasive surgeries in the treatment of laryngeal cancer, but some patients still have recurrence after operations (6), which seriously affects their health.

MicroRNA (miRNA) is a non-protein-encoded microRNA, with similar length to 18-25 nucleotides. It acts on messenger RNA to degrade miRNA or prevents its transcription, translation and regulate its gene expression; since miRNAs were discovered, many studies have confirmed that they can facilitate the expression of protooncogenes or inhibit the expression of tumor suppressor genes (7). Further studies have shown that miRNA plays an extremely important role in many biological aspects, including cell metabolism, cell proliferation, apoptosis, cell development and cell differentiation (8). With certain tissue specificity, miRNA is an ideal cancer biomarker and therapeutic monitoring indicator and its expression has important diagnostic value in colon, breast, and gastric cancer 
Table I. Primer sequences of miRNA-145, miRNA-218 and internal reference U6.

\begin{tabular}{lll}
\hline Group & \multicolumn{1}{c}{ Upstream primer } & \multicolumn{1}{c}{ Downstream primer } \\
\hline miRNA-145 & 5'-GTCCAGTTTTCCCAGGAAT-3' & 5'-TGGTGTCGTGGAGTCG-3' \\
miRNA-218 & 5'-GCGCTTGTGCTTGATCTAA-3' & 5'-GTGCAGGGTCCGAGGT-3' \\
U6 & 5'-CTCGCTTCGGCAGCACATATACT-3' & 5'-ACGCTTCACGAATTTGCGTGTC-3'
\end{tabular}

and hepatocellular carcinoma, and the expression in laryngeal cancer is significantly different from that in adjacent normal tissues (8).

miRNA-145 is one of the important tumor suppressors, and its expression level is decreased in some types of cancer, such as gallbladder, colorectal, prostate cancer and pleural mesothelioma. Studies have shown that miRNA-145 can inhibit the proliferation and metastasis of laryngeal cancer epithelial cells. The decrease of the expression level of miRNA-145 can lead to poor prognosis of patients with laryngeal cancer (9). miRNA-218 has a function of inhibiting tumors during the development, studies have manifested that the increase of the expression level of miRNA-218 can inhibit the proliferation and metastasis of cancer cells. There are different degrees of decrease in the expression level of this gene in kidney, gastric cancer and nasopharyngeal cancer (10).

Previous reports are few on the expression of miRNA-145 and miRNA-218 in patients with laryngeal cancer and the relationship among the two genes, clinicopathology and prognosis. The present study investigated the expression levels of miRNA-145 and miRNA-218 and the relationship among the two genes, clinicopathology and prognosis by observing the expression of miRNA-145 and miRNA-218 in serum of patients with laryngeal cancer to provide a theoretical basis in clinic.

\section{Patients and methods}

General data. The clinical medical records of 132 patients with laryngeal cancer admitted to the Guangdong Second Provincial General Hospital (Guangzhou, China) from February 2009 to March 2014 were retrospectively analyzed in this study. They were the study group, in which there were 85 males and 47 females with an average age of $63.35 \pm 12.45$ years. In addition, the data of 56 healthy volunteers who took physical examinations in Guangdong Second Provincial General Hospital were collected. They comprised the control group, in which there were 37 males and 19 females with an average age of $63.01 \pm 11.94$ years.

This study was approved by the Ethics Committee of Guangdong Second Provincial General Hospital. The subjects and their family members were informed and signed the informed consent form.

Inclusion and exclusion criteria. Inclusion criteria were: i) age $\geq 18$ years; ii) the absence of radiotherapy, chemotherapy or any adjuvant therapy before hospitalization, the absence of other malignant tumors; iii) the absence of a history of family genetic diseases; and iv) regular return visits after hospital discharge.
Exclusion criteria were: i) the presence of antibiotics taking within three months before blood collection; ii) the presence of liver insufficiency; iii) the presence of autoimmune system deficiency; iv) laryngeal cancer was recurrent during hospitalization; v) the presence of upper respiratory tract infection; and vi) the incompleteness of clinicopathologic data.

\section{Experiment methods}

Experiment instruments and reagents. miRcute serum miRNA Extraction and Separation kit (model: DP503; Beijing Tiangen Biotech Co., Ltd., Beijing, China); miRcute enhanced miRNA cDNA First-Strand Synthesis kit (model no. KR211; Beijing Tiangen Biochemical Technology Co., Ltd.); high-speed centrifuge (model no. HC-2518R; Anhui USTC Zonkia Scientific Instrument Co., Ltd., Hefei, China); 7500 Real-Time PCR System fluorescence quantitative instrument (model no. 7500 Fast; Applied Biosystems; Thermo Fisher Scientific, Inc., Waltham, MA, USA); miRcute enhanced miRNA fluorescence quantitative test kit (article no. FP411-02; Beijing Tiangen Biochemical Technology Co., Ltd.) were used in the prsent study. Primer sequences of miRNA-145, miRNA-218 and internal reference U6 were designed and produced by Beijing Tiangen Biochemical Technology Co., Ltd., and are shown in Table I.

Serum collection. A sterile pro-coagulation tube was used to collect $4 \mathrm{ml}$ of the fresh blood of the study group and the control group, and then it was left at room temperature for $30 \mathrm{~min}$. The serum was separated in a centrifuge at a speed of $2,800 \times \mathrm{g}$ for approximately $10 \mathrm{~min}$ at $4^{\circ} \mathrm{C}$. Next, a $2 \mathrm{ml}$ RNase-free EP tube was used to collect the supernatant and all the specimens were placed in a refrigerator at $-80^{\circ} \mathrm{C}$.

Extraction of total RNA and reverse transcription synthesis of $c D N A$. Frozen serum specimens were left at room temperature for $30 \mathrm{~min}$. Lysate solution $(900 \mu \mathrm{l})$ was added into $200 \mu \mathrm{l}$ of the serum, and then was oscillated and mixed using a shaker for $30 \mathrm{sec}$ until complete homogenate was finished. Next, the mixture was left at room temperature for $5 \mathrm{~min}$. After protein nucleic acid compound was completely separated, $200 \mu \mathrm{l}$ of chloroform was added into the mixture and it was violently oscillated for $15 \mathrm{sec}$. After oscillation, it was placed at room temperature for $5 \mathrm{~min}$, and then it was centrifuged at $10,000 \mathrm{x} \mathrm{g}$ for $15 \mathrm{~min}$ at $4^{\circ} \mathrm{C}$. The top colorless aqueous phase was carefully collected and transferred to a new tube and $1 \mathrm{ml}$ of absolute ethanol was added and mixed. Then it was centrifuged for $30 \mathrm{sec}$ according to the aforementioned step. Next, $500 \mu 1$ of deproteinized MRD was added, and the mixture was centrifuged again for $30 \mathrm{sec}$. Spent liquor was discarded and the adsorption column, miRelute was transferred to a new RNase-free centrifuge tube with a volume of $1.5 \mathrm{ml}$. 
Table II. Comparison of the general baseline data between the study group and the control group [n, (\%)] (mean \pm SD).

\begin{tabular}{|c|c|c|c|c|}
\hline Category & Study group $(\mathrm{n}=132)$ & Control group $(\mathrm{n}=56)$ & $\chi^{2 / t}$ & P-value \\
\hline Age (years) & $63.35 \pm 12.45$ & $63.01 \pm 11.94$ & 0.173 & 0.863 \\
\hline Sex & & & 0.049 & 0.869 \\
\hline Male & 85 (64.39) & $37(66.07)$ & & \\
\hline Female & $47(35.61)$ & $19(33.93)$ & & \\
\hline Smoking & & & 0.033 & 0.871 \\
\hline Yes & $82(62.12)$ & $34(60.71)$ & & \\
\hline No & $50(37.88)$ & $22(39.29)$ & & \\
\hline Body mass index $\left(\mathrm{kg} / \mathrm{m}^{2}\right)$ & & & 0.394 & 0.627 \\
\hline$<24$ & $76(57.58)$ & $35(62.50)$ & & \\
\hline$\geq 24$ & $56(42.42)$ & $21(37.50)$ & & \\
\hline Heart rate (time/min) & & & 0.865 & 0.346 \\
\hline$<60$ & $7(5.30)$ & $5(8.93)$ & & \\
\hline$\geq 60$ & $125(94.70)$ & $51(91.07)$ & & \\
\hline Marriage history & & & 0.527 & 0.488 \\
\hline Married & $126(95.45)$ & $52(92.86)$ & & \\
\hline Not married & $6(4.55)$ & $4(7.14)$ & & \\
\hline Fasting blood glucose level (mmol/l) & $4.51 \pm 0.58$ & $4.58 \pm 0.61$ & 0.745 & 0.457 \\
\hline $\mathrm{Hb}(\mathrm{g} / \mathrm{l})$ & $121.02 \pm 11.32$ & $117.99 \pm 12.78$ & 1.614 & 0.108 \\
\hline $\operatorname{RBC}\left(\times 10^{12} / 1\right)$ & $4.48 \pm 0.28$ & $4.52 \pm 0.31$ & 0.867 & 0.387 \\
\hline $\operatorname{PLT}\left(\mathrm{x} 10^{9} / \mathrm{l}\right)$ & $219.28 \pm 35.98$ & $216.12 \pm 38.71$ & 0.538 & 0.591 \\
\hline
\end{tabular}

Hb, hemoglobin; RBC, red blood cell; PLT, platelet.

Subsequently, $20 \mu \mathrm{l}$ of RNase-free $\mathrm{ddH}_{2} \mathrm{O}$ was added, after it was left for $2 \mathrm{~min}$, it was centrifuged at $10,000 \mathrm{x}$ for $2 \mathrm{~min}$ at $4^{\circ} \mathrm{C}$, and total RNA was obtained. Agarose gel electrophoresis (1\%) was used to test the integrity of RNA and the qualified RNA samples were selected to synthesize cDNA, while $1 \mu \mathrm{l}$ of total RNA was used to reverse transcribe cDNA. The thawed $2 \mathrm{X}$ miRNA RT reaction buffer was mixed fully, and the total volume of miRNA RT enzyme was $20 \mu \mathrm{l}$. A pipette was used to mix the aforementioned prepared reaction solution to synthesize cDNA, and the reaction was carried out at $42^{\circ} \mathrm{C}$ for $60 \mathrm{~min}$.

Detection of the expression levels of miRNA-145 and miRNA-218. RT-qPCR was used to detect the expression levels of miRNA-145 and miRNA-218 of the two groups. A total of $20 \mu \mathrm{l}$ of reaction system was prepared, it was predenatured at $95^{\circ} \mathrm{C}$ for $15 \mathrm{~min}$, and then denatured at $95^{\circ} \mathrm{C}$ for $20 \mathrm{sec}$; at $64^{\circ} \mathrm{C}$ for $30 \mathrm{sec}$, at $72^{\circ} \mathrm{C}$ for $34 \mathrm{sec}, 42$ cycles were made, and finally it was annealed at $60^{\circ} \mathrm{C}$ for $34 \mathrm{sec}$. The standard curve was drawn according to the concentration of the standard sample and the average value of the cyclic thresholds corresponding to each concentration. According to the standard curve, the instrument automatically calculated the copy numbers $(/ \mathrm{ml})$ of miRNA-145, miRNA-218 and internal reference U6 in serum, while the expression of miRNA-145 and miRNA-218 was reflected by the ratio of miRNA-145, miRNA-218 and U6.

Follow up of the study group. A 48-month follow up was carried out on the patients in the study group, who were discharged from the hospital. Telephone interviews were conducted. The patients' survival condition was recorded in a timely manner once a month after discharge from the hospital. For patients with poor physical condition and poor communication, telephone interview was not suitable. If considered necessary their home was visited or their relatives consulted, to ensure the accuracy of the experiment.

Statistical analysis. In the present study, SPSS 17.0 software package (Beijing Strong-Vinda Information Technology Co., Ltd., Beijing, China) was used to statistically analyze the collected data and obtain images. The measurement data were expressed in the form of average value \pm standard deviation (mean $\pm \mathrm{SD}$ ). A Student's t-test was used to compare the measurement data between the groups and the Chi-square test was used to compare the enumeration data between the groups. Kaplan-Meier was used to carry out the survival analysis together with the log-rank test. $\mathrm{P}<0.05$ indicated differences were statistically significant.

\section{Results}

General baseline data. Age, sex, body mass index, heart rate, marriage history, smoking, fasting blood glucose level, hemoglobin $(\mathrm{Hb})$ value, red blood cell (RBC) value, and platelet (PLT) value of the study group and the control group were compared, and differences were not statistically significant (P>0.05) (Table II). 
Table III. Relationship between the expression of miRNA-145 and the clinicopathological characteristics of the study group.

\begin{tabular}{|c|c|c|c|c|}
\hline Category & $\mathrm{N}$ & $\begin{array}{l}\text { Study } \\
\text { group } \\
(\mathrm{n}=132)\end{array}$ & $\mathrm{t}$ & P-value \\
\hline Age (years) & & & 1.780 & 0.077 \\
\hline$<60$ & 48 & $1.79 \pm 0.41$ & & \\
\hline$\geq 60$ & 84 & $1.92 \pm 0.40$ & & \\
\hline Sex & & & 1.161 & 0.248 \\
\hline Male & 85 & $1.81 \pm 0.43$ & & \\
\hline Female & 47 & $1.90 \pm 0.42$ & & \\
\hline Body mass index $\left(\mathrm{kg} / \mathrm{m}^{2}\right)$ & & & 1.489 & 0.139 \\
\hline$<24$ & 76 & $1.82 \pm 0.44$ & & \\
\hline$\geq 24$ & 56 & $1.93 \pm 0.39$ & & \\
\hline Degree of differentiation & & & 13.200 & $<0.01$ \\
\hline Middle, low & 75 & $1.62 \pm 0.24$ & & \\
\hline High & 57 & $2.13 \pm 0.19$ & & \\
\hline Lymph node metastasis & & & 8.227 & $<0.01$ \\
\hline Yes & 74 & $1.67 \pm 0.29$ & & \\
\hline No & 58 & $2.07 \pm 0.25$ & & \\
\hline Tumor diameter (cm) & & & 10.460 & $<0.01$ \\
\hline$<5$ & 48 & $2.11 \pm 0.21$ & & \\
\hline$\geq 5$ & 84 & $1.66 \pm 0.28$ & & \\
\hline TNM staging & & & 8.972 & $<0.01$ \\
\hline I-II & 54 & $2.08 \pm 0.24$ & & \\
\hline III-IV & 78 & $1.66 \pm 0.28$ & & \\
\hline
\end{tabular}

Expression levels of miRNA-145 and miRNA-218 in the two groups. The results showed that the expression level of miRNA-145 in the study group was $1.85 \pm 0.47$, which was lower than that in the control group, while the expression level of miRNA-145 in the control group was 5.73 \pm 0.93 , the difference was statistically significant $(\mathrm{t}=37.930, \mathrm{P}<0.01)$. The expression level of miRNA-218 in the study group was $3.15 \pm 0.95$, which was lower than that in the control group, while the expression level of miRNA-218 in the control group was $8.73 \pm 2.84$, the difference was statistically significant $(\mathrm{t}=20.130, \mathrm{P}<0.01)$ (Fig. 1).

Relationship between the expression of miRNA-145 and the clinicopathological characteristics of the study group. The results showed that the expression level of miRNA-145 in the study group was not related to age, sex and body mass index $(\mathrm{P}>0.05)$. The expression level of miRNA-145 was related to the degree of differentiation, lymph node metastasis, tumor diameter and TNM staging $(\mathrm{P}<0.05)$ (Table III).

Relationship between the expression of miRNA-218 and the clinicopathological characteristics of the study group. The results showed that the expression level of miRNA-218 in the study group was not related to age, sex and body mass index ( $P>0.05)$; the expression level of miRNA-218 was related to the degree of differentiation, lymph node metastasis, tumor diameter and TNM staging $(\mathrm{P}<0.05)$ (Table IV).
Table IV. Relationship between the expression of miRNA-218 and the clinicopathological characteristics of the study group.

\begin{tabular}{|c|c|c|c|c|}
\hline Category & $\mathrm{N}$ & $\begin{array}{l}\text { Study } \\
\text { group } \\
(\mathrm{n}=132)\end{array}$ & $\mathrm{t}$ & P-value \\
\hline Age (years) & & & 0.811 & 0.419 \\
\hline$<60$ & 48 & $3.22 \pm 0.88$ & & \\
\hline$\geq 60$ & 84 & $3.09 \pm 0.89$ & & \\
\hline Sex & & & 0.932 & 0.353 \\
\hline Male & 85 & $3.19 \pm 0.91$ & & \\
\hline Female & 47 & $3.04 \pm 0.84$ & & \\
\hline
\end{tabular}

Body mass index $\left(\mathrm{kg} / \mathrm{m}^{2}\right)$

$\begin{array}{lll}<24 & 76 & 3.07 \pm 0.87 \\ \geq 24 & 56 & 3.18 \pm 0.92\end{array}$

Degree of differentiation

Middle, low

High

$0.701 \quad 0.485$

$56 \quad 3.18 \pm 0.92$

Lymph node metastasis

Yes

No

$\begin{array}{ll}74 & 2.97 \pm 0.77\end{array}$

$58 \quad 3.82 \pm 0.28$

Tumor diameter $(\mathrm{cm})$

$<5$

$\geq 5$

$\begin{array}{ll}75 & 2.78 \pm 0.58 \\ 57 & 3.69 \pm 0.41\end{array}$

$57 \quad 3.69 \pm 0.41$

$7.997<0.01$

TNM staging

I-II
$8.615<0.01$

$48 \quad 3.67 \pm 0.43$

$84 \quad 2.81 \pm 0.61$

$8.558<0.01$

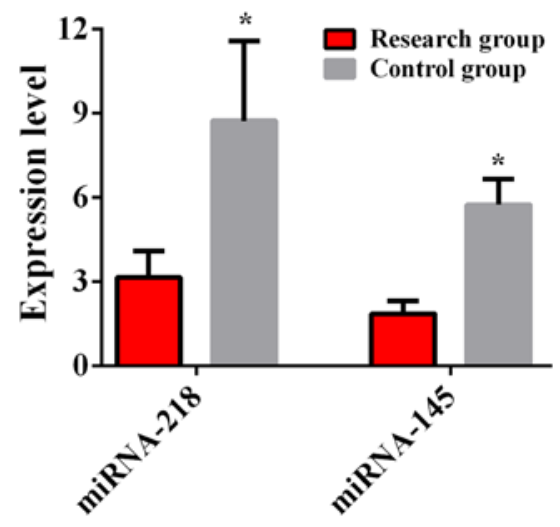

Figure 1. Comparison of the expression levels of miRNA-145 and miRNA-218 between the study and control groups. The detection results of RT-qPCR show that the expression levels of miRNA-145 and miRNA-218 in serum of the study group are lower than those of the control group, and the differences are statistically significant $(\mathrm{P}<0.05) .{ }^{*} \mathrm{P}<0.05$, the differences are statistically significant compared to the research group.

Comparison of the survival condition in the study group. According to the relative expression levels of miRNA-145 and miRNA-218 on the day when the patients of the study group were discharged from the hospital, the study group was divided into the high expression group and the low expression group. There were 73 patients in the high expression group 


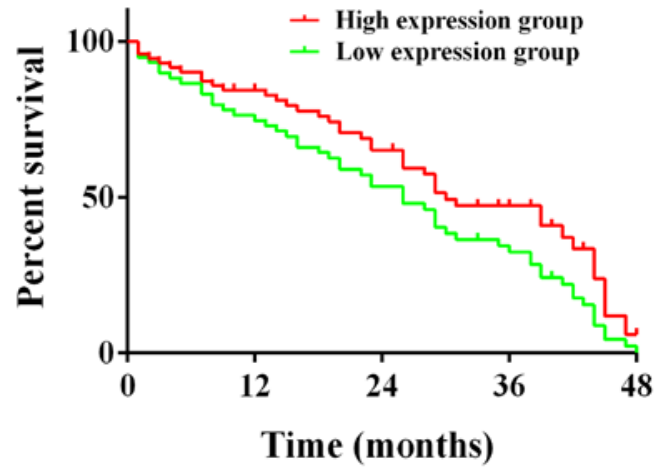

Figure 2. Comparison of the survival condition among the miRNA-145 high expression group, the miRNA-145 low expression group, the miRNA-218 high expression group and the miRNA-218 low expression group. The detection results of Kaplan-Meier and log-rank test show that the median survival time of the high expression group was 30 months, and the median survival time of the low expression group was 26 months. At the end of the return visit, the mortality of the high expression group was $54.79 \%$, and the mortality of the low expression group was $89.83 \%$, the differences between the two groups are statistically significant $\left(\chi^{2}=4.371, \mathrm{P}=0.037\right)$.

and 59 patients in the low expression group. A return visit was carried out for the patients in the two groups at 48 months. At the end of the return visit, 40 patients who were in the high expression group had died, the median survival time was 30 months and the mortality was $54.79 \%$. Fifty-three patients who were in the low expression group had died, the median survival time was 26 months, the mortality was $89.83 \%$. The difference between the two groups was statistically significant $\left(\chi^{2}=4.371, \mathrm{P}=0.037\right)($ Fig. 2$)$.

\section{Discussion}

Laryngeal cancer is a malignant tumor of the epithelium in laryngeal mucosa. It frequently appears in male and is a common head cancer, accounting for approximately $25 \%$ of head and neck cancers (11). Laryngeal cancer can occur in any part of the larynx, including supraglottic portion, infraglottic portion, and glottic portion. In recent years, the morbidity of laryngeal cancer has gradually increased. There are approximately 157,000 new cases of laryngeal cancer in the world annually. Conventional treatment of advanced laryngeal cancer is mainly surgical treatment, including total laryngectomy and partial laryngectomy (12). In recent years, with the advancement of technology, both surgery and radiotherapy have made significant progress, but surgical treatment, radiotherapy and chemotherapy have devastating effects on language function and swallowing and eating function of patients. The survival condition of patients with advanced laryngeal cancer is poor. The 5-year survival rate of patients with laryngeal cancer has slightly improved compared with past decades. The patients' local recurrence rate is increased $(8,13)$. Therefore, it is imperative and necessary to better understand the potential molecular mechanism and develop further effective prognostic evaluation factors and therapeutic targets.

It is well known that miRNAs play an important role in various human cancers and act as an oncogene or a tumor suppressor gene. Findings have shown that the abnormal expression of miRNA is related to many human diseases, especially cancer. miRNA microarray detection confirms that miRNA which is expressed abnormally may potentially serve in diagnosing cancers and may be used as a prognostic biomarker of patients with cancer, thereby playing an important role in the pathogenesis of cancer (13). miRNA-145 is located on chromosome 5 in human body, and $5 \mathrm{q} 33$ is a highly conserved, non-coding, and singlestranded microRNA (14). Previous findings have shown that miRNA-145 is abnormally expressed in colorectal cancer. The expression of miRNA-145 in various colorectal cancer cells is lower than that in normal large intestine epithelial cells, and the higher the metastatic potential of cancer cells is, the lower the expression is (15). Additionally, ectopic overexpression of miRNA-145 can inhibit the proliferation and invasion in vitro of tumor cells, while stable expression of miRNA-145 can inhibit growth and metastasis of tumors in large intestine models of nude mouse (15). Human mature miRNA-218 has two different coding genes, miRNA-218-1 and $m i R N A-218-2$ which are, respectively, located on Slit 2 gene of human chromosome 4p15.31 and Slit3 gene of 6q35.1. It has been clarified that the decrease of the expression level of miRNA-218 in cancer cells is caused by hypermethylation of Slit2 gene promoter and Slit3 gene promoter (16). The expression level of miRNA-218 decreases in many human cancer types, such as head and neck squamous cell, non-small cell lung, pancreatic ductal and gastric cancer. It has been proven that miRNA-218 can inhibit the migration and invasion of some cancer cells, including gastric, glioma, and cervical cancer and that in cervical cancer, the expression level of miRNA-218 is related to lymph node metastasis of cancer cells (17).

The expression levels of miRNA-145 and miRNA-218 in serum of the patients with laryngeal cancer and the volunteers who took normal physical examination were detected in this study, and it was found that the expression levels of miRNA-145 and miRNA-218 in serum of the patients with laryngeal cancer were lower than those of the volunteers who took normal physical examination, with the differences being statistically significant $(\mathrm{P}<0.05)$. This result is similar to that of Karatas et al who initially found that the expression level of miRNA-145 decreased in laryngeal cancer tissue (18). Further studies found that miRNA-145 exerted an inhibitory effect on laryngeal cancer stem cell markers, such as SOX2, OCT4, KLF4 and ABCG2. In the study of Cheng et al, it is considered that miRNA-218 expression is low in bladder cancer, whereas a high expression of miRNA-218 can inhibit growth, proliferation and migration of cancer cells, and miRNA-218 facilitates an increase of the expression levels of tumor-inhibiting factor-protein-phosphatase and tensin 10 chromosome homologous region genes tumor by indirectly inhibiting BMI-1 (19). Feng et al (20) found that the expression level of miRNA-218 in laryngeal cancer tissue was significantly lower than that in adjacent normal tissue, and that miRNA-218 that was highly expressed inhibited proliferation and invasion of laryngeal cancer cells. The expression levels of miRNA-145 and miRNA-218 in serum of patients with laryngeal cancer were related to the degree of differentiation, lymph node metastasis, tumor diameter and TNM staging, and the differences were statistically significant $(\mathrm{P}<0.05)$. According to the expression levels of miRNA-145 and miRNA-218 on 
the day when the patients in the study group were discharged from the hospital. The study group was divided into two groups, miRNA-145 and miRNA-218 in serum of 73 patients were, respectively, 3.00 or $>3.00$ and 5.00 or $>5.00$, and those patients were considered the high expression group. miRNA-145 and miRNA-218 in serum of 59 patients were, respectively, $<3.00$ and $<5.00$, and those patients were considered the low expression group. A return visit was carried out for the patients in the two groups, at 48 months. At the end of the return visit, 40 patients who were in the high expression group had died, the median survival time was 30 months and the mortality was $54.79 \%$; and 53 patients who were in the low expression group had died, the median survival time was 26 months and the mortality was $89.83 \%$. The differences between the two groups were statistically significant $\left(\chi^{2}=4.371\right.$, $\mathrm{P}=0.037)$. Karatas et al considered that the expression level of miRNA-145 played a key role in cancer cell metastasis, tumor staging, tumorigenesis and prognosis of patients with laryngeal cancer (21). This result is similar to the results of the present study. The study of Zhao et al suggested that miRNA-145 expression in patients with laryngeal cancer was low (22). The expression level of miRNA-145 was negatively correlated with the expression level of MYO5A. miRNA-145 directly regulated the expression of MYO5A, inhibited proliferation and invasion of laryngeal cancer cells and facilitated apoptosis of laryngeal cancer cells by inhibiting the expression level of MYO5A, and that the increase of the expression level of MYO5A in serum of patients with laryngeal cancer could lead to lymph node metastasis and poor prognosis of patients. Xin et al (23) found that the expression level of miRNA-218 was closely related to staging, grading and metastasis of gastric cancer. The low expression of miRNA-218 could lead to an increase in the grade of cancer staging of patients with gastric cancer, 3-year survival rate of gastric cancer patients with high expression of miRNA-218 was higher than that of gastric cancer patients with low expression of miRNA-218. miRNA-218 was a predictive indicator of prognosis of patients with gastric cancer. Thus, a decrease of the expression levels of miRNA-145 and miRNA-218 may have a negative impact on survival conditions of patients with laryngeal cancer.

Retrospective case analysis and research were used in this study. The cases were selected strictly according to the inclusion and exclusion criteria. The basic data investigation, clinical examination and special examination of the patients were scrupulously recorded and arranged, ensuring the reliability of the study results. As for the post-operative return visit for the patients with laryngeal cancer, a 48-month return visit was carried out and monthly telephone interviews, home visits, or interviewing relatives of the patients were carried out to ensure more accurate and authentic study results.

The expression levels of miRNA-145 and miRNA-218 decrease in serum of patients with laryngeal cancer. The expression levels of miRNA-145 and miRNA-218 are related to the degree of differentiation, lymph node metastasis, tumor diameter and TNM staging. The higher the expression levels of miRNA-145 and miRNA-218 in patients with laryngeal cancer are, the better the prognosis is, and the longer the survival time is. miRNA-145 and miRNA-218 can be used as indicators to evaluate the prognosis of patients with laryngeal cancer, which is worth implementing in clinic.

\section{Acknowledgements}

Not applicable.

\section{Funding}

No funding was received.

\section{Availability of data and materials}

The datasets used and/or analyzed during the present study are available from the corresponding author on reasonable request.

\section{Authors' contributions}

LG wrote the manuscript and helped with extraction of total RNA and reverse transcription synthesis of cDNA. XC and WHu analyzed and interpreted the patients' general data. WHua and WY performed the PCR. YL, SY and YC were responsible for the observation indicators analysis. All authors read and approved the final manuscript.

\section{Ethics approval and consent to participate}

The present study was approved by the Ethics Committee of Guangdong Second Provincial General Hospital (Guangzhou, China). Patients who participated in this research had complete clinical data. Signed informed consents were obtained from the patients or the guardians.

\section{Patient consent for publication}

Not applicable.

\section{Competing interests}

The authors declare that they have no competing interests.

\section{References}

1. Emadzadeh M, Shahidsales S, Mohammadian Bajgiran A, Salehi M, Massoudi T, Nikfarjam Z and Salehi M: Head and neck cancers in North-East Iran: A 25 year survey. Iran J Otorhinolaryngol 29: 137-145, 2017.

2. Genç R, Çă̆lı S, Yüce İ, Vural A, Okuducu H, Patıroğlu T and Güney E: The role of $H$. pylori in the development of laryngeal squamous cell carcinoma. Dis Markers 35: 447-449, 2013.

3. Wang S, Wu J, Song Y and Zhong H: Expression of endothelin-1 in laryngocarcinoma tissues and its clinical significance. Oncol Lett 11: 3366-3368, 2016

4. Haws L Jr and Haws BT: Aerodigestive cancers: Laryngeal cancer. FP Essent 424: 26-31, 2014.

5. Setiawan IGB and Adiputra PAT: A successful tracheal resection and anastomosis in papillary thyroid carcinoma with tracheal invasion. Open Access Maced J Med Sci 6: 2161-2164, 2018.

6. Yu X, Ma C, Fu L, Dong J and Ying J: MicroRNA-139 inhibits the proliferation, migration and invasion of gastric cancer cells by directly targeting $\mathbf{0}$-associated protein kinase 1 . Oncol Lett 15: 5977-5982, 2018.

7. Seux M, Iovanna J, Dagorn JC and Dusetti NJ: MicroRNAs in pancreatic ductal adenocarcinoma: New diagnostic and therapeutic clues. Pancreatology 9: 66-72, 2009.

8. Alkhubaizi Q, Khalaf ME, Dashti H and Sharma PN: Oral cancer screening among smokers and nonsmokers. J Int Soc Prev Community Dent 8: 553-559, 2018. 
9. Ding Y, Wu Y, Gao W, Zhang C, Zhao Q, Guo H, Qu X, Wen S and Wang B: Analysis of gene expression profiling variations induced by hsa-miR-145-5p-overexpression in laryngeal squamous cell carcinoma cell line Tu-177. Mol Med Rep 16: 5863-5870, 2017.

10. Elser H, Falconi AM, Bass M and Cullen MR: Blue-collar work and women's health: A systematic review of the evidence from 1990 to 2015. SSM Popul Health 6: 195-244, 2018.

11. Citil S, Dogan S, Atilgan HI, Menzilcioglu MS, Sahin T, Abdulrezzak U, Duymus M and Ozturk M: Comparison of dynamic contrast-enhanced MRI and PET/CT in the evaluation of laryngeal cancer after inadequate CT results. Pol J Radiol 80: 428-432, 2015

12. Hamilton NJI and Birchall MA: Tissue-engineered larynx Future applications in laryngeal cancer. Curr Otorhinolaryngol Rep 5: 42-48, 2017.

13. Yu X and Li Z: The role of microRNAs expression in laryngeal cancer. Oncotarget 6: 23297-23305, 2015.

14. Farrokhnia F, Aplin JD, Westwood M and Forbes K: MicroRNA regulation of mitogenic signaling networks in the human placenta. J Biol Chem 289: 30404-30416, 2014.

15. Feng Y, Zhu J, Ou C, Deng Z, Chen M, Huang W and Li L: MicroRNA-145 inhibits tumour growth and metastasis in colorectal cancer by targeting fascin-1. Br J Cancer 110 : 2300-2309, 2014.

16. Alajez NM, Lenarduzzi M, Ito E, Hui AB, Shi W, Bruce J, Yue S, Huang $\mathrm{SH}, \mathrm{Xu} \mathrm{W}$, Waldron J, et al: MiR-218 suppresses nasopharyngeal cancer progression through downregulation of survivin and the SLIT2-ROBO1 pathway. Cancer Res 71: 2381-2391, 2011.

17. Misawa K, Imai A, Mochizuki D, Mima M, Endo S, Misawa Y, Kanazawa T and Mineta H: Association of TET3 epigenetic inactivation with head and neck cancer. Oncotarget 9: 24480-24493, 2018.
18. Karatas OF, Yuceturk B, Suer I, Yilmaz M, Cansiz H, Solak M, Ittmann M and Ozen M: Role of miR-145 in human laryngeal squamous cell carcinoma. Head Neck 38: 260-266, 2016.

19. Cheng Y, Yang X, Deng X, Zhang X, Li P, Tao J and Lu Q MicroRNA-218 inhibits bladder cancer cell proliferation, migration, and invasion by targeting BMI-1. Tumour Biol 36: 8015-8023, 2015.

20. Feng J, Fan Y, Ayiheng Q, Zhang H, Yong J and $\mathrm{Hu} B$ MicroRNA-125b targeted STAT3 to inhibit laryngeal squamous cell carcinoma cell growth and motility. Oncol Lett 14: 480-486, 2017.

21. Karatas OF, Suer I, Yuceturk B, Yilmaz M, Hajiyev Y, Creighton CJ, Ittmann M and Ozen M: The role of miR-145 in stem cell characteristics of human laryngeal squamous cell carcinoma Hep-2 cells. Tumour Biol 37: 4183-4192, 2016.

22. Zhao X, Zhang W and Ji W: MYO5A inhibition by miR-145 acts as a predictive marker of occult neck lymph node metastasis in human laryngeal squamous cell carcinoma. Onco Targets Ther 11: 3619-3635, 2018.

23. Xin SY, Feng XS, Zhou LQ, Sun JJ, Gao XL and Yao GL: Reduced expression of circulating microRNA-218 in gastric cancer and correlation with tumor invasion and prognosis. World J Gastroenterol 20: 6906-6911, 2014 International (CC BY-NC-ND 4.0) License. 